\title{
A Stochastic Model for Estimating the Statistical Measures for Time to Cross the Antigenic Diversity Threshold of HIV Infected Under Correlated Inter-Contact Times using Erlang Truncated Exponential Distribution
}

\author{
R. Kannan ${ }^{1}$, G. Parthasarathi ${ }^{2^{*}}$ \\ ${ }^{1,2}$ Dep. of Statistics, Annamalai University, Tamil Nadu, India \\ *Corresponding Author: pgstat.hct@gmail.com, Tel.: +919600131084
}

Available online at: www.isroset.org

Accepted 10/Aug/2018, Online 30/Aug/2018

\begin{abstract}
This study is based on stochastic model for estimating the seroconversion time of HIV transmission under correlated inter- contact times. In the estimation of expected time to seroconversion, there is an important role for the inter-arrival times between successive contacts and has a significant influence. In this paper, it is assumed that the inter contact times between successive contacts are correlated random variables and the threshold distribution as Erlang truncated exponential distribution. In developing this model the result of Gurland (1995) has been used. The mean time to seroconversion and its variance are derived and the numerical illustrations are provided.
\end{abstract}

Keywords- Human Immune deficiency virus, Acquired Immune Deficiency Syndrome, Intercontact times, Seroconversion, Antigenic Diversity threshold.

\section{INTRODUCTION}

In the study of HIV infection and its progression, the antigenic diversity of the antigen namely the HIV plays an important role. The intensity of sexual contact is an important factor that adds to the antigenic diversity. Since more number of new antigens are acquired by the individual who is getting infected. The process of divergence of the antigenic properties of the invading antigens requires a constant monitoring, so that the preventive strategies to arrest the growth of antigenic diversity can be adopted. The intercontact times do play a role in the increase of antigenic diversity. The antigenic diversity model has been discussed by Nowak and May (1991) and stilianakis et.al.(1994).

In the estimation of expected time to seroconversion, there is an important role for the inter-arrival times between successive contacts and it has a significant influence. Sathiyamoorthi and Kannan (2006) and Kannan et al.(2007) have obtained the expected time to seroconversion under the assumption that the inter-arrival times between the contacts are not independent but constantly correlated. In this paper a stochastic model assumes inter-contact times between successive contacts are correlated random variables and the threshold distribution which follows Erlang truncated exponential distribution. These assumption of correlated inter-contact times seems plausible by the fact that any partner after every contact with an index may have a physiological obsession and fear of contracting the disease, which may have an impact on inter-contact times of contacts such as prolongation of inter-contact times.

In developing such a stochastic model the shock model and cumulative damage process discussed by Esary et al.(1973). is used. Shock model with correlated inter-contact times has been studied by the Sathiyamoorthi (1979). In developing this model the results of the Gurland (1955) has been used. Using the same concept, expected time to seroconversion and its variance are obtained in this paper. In this study the theoretical results are substantiated using numerical data simulated.

\section{ASSUMPTIONS OF THE MODEL}

(i) Human Immuno deficiency Virus transmits only through sexual contacts.

(ii) An uninfected individual has sexual inter-group communication with a Human Immuno deficiency virus infected individual and a random amount of HIV getting transmitted at each contacts. 
(iii) An individual damagae process is assumed to be linear and cumulative in the immune system.

(iv) In each contact, the transmission of HIV will damage to the individual's immune system and time intervals between each successive contact are assumed to be correlated.

(v) The procedure that generates the contacts and also the sequence of damages to the threshold are mutually independent.

(vi) The total damage caused once exceeds a threshold level $\mathrm{Y}$ that it could be a random variable and it leads to sero-conversion and an individual is considered a sero positive.

\section{NotATIONS}

$\mathrm{X}_{\mathrm{i}} \quad$ : a random variable denoting the increase in the antigenic diversity arising due to the HIV transmitted during the $i^{\text {th }}$ contact $\mathrm{X}_{1}, \mathrm{X}_{2}, \ldots \mathrm{X}_{\mathrm{k}}$ are continuous i.i.d. random variables, with p.d.f. g(.) and c.d.f. G(.).

Y : a random variable representing antigenic diversity threshold and follows erlang truncated exponential distribution with parameters $\theta$

and $\lambda$, the p.d.f being $h($.$) and c.d.f H($.$) .$

$\mathrm{U}_{\mathrm{i}} \quad$ : a continuous random variable denoting the interarrival times between successive contacts with p.d.f.f(.) and c.d.f. $F($.$) .$ $\mathrm{g}_{\mathrm{k}}($.$) \quad : The p.d.f of random variable \sum_{i=1}^{k} X_{i}$

$\mathrm{F}_{\mathrm{k}}() \quad:$.$\quad The \mathrm{k}^{\text {th }}$ convolution of $\mathrm{F}($.$) .$

$\mathrm{T}$ : A continuous random variable denoting the time of seroconversion with p.d.f. $l($.$) and c.d.f. L(.).$

$\mathrm{V}_{\mathrm{k}}(\mathrm{t})$ : The probability of exactly k contacts in $(\mathrm{o}, \mathrm{t}]$.

$l^{*}(\mathrm{~s}) \quad: \quad$ The Laplacestieltje'stransform of $l(\mathrm{t})$.

$f *(\mathrm{~s}) \quad$ : The Laplacestieltje'stransform of $f(\mathrm{t})$.

$\rho$ : the correlation between $\mathrm{Xi}$ and $\mathrm{Xj}, \mathrm{i} \neq \mathrm{j}$.

$$
Z_{k}=\sum_{i=1}^{k} U_{i}
$$

It can be shown that

\section{RESULTS}

$$
\mathrm{P}\left[\sum_{\mathrm{i}=1}^{\mathrm{k}} \mathrm{X}_{\mathrm{i}}<\mathrm{Y}\right]=\int_{0}^{\infty} \mathrm{g}_{\mathrm{k}}(x) \overline{\mathrm{H}}(x) \mathrm{d} x
$$

Where $\overline{\mathrm{H}}(x)=1-\mathrm{H}(x)$

Let $\mathrm{Y} \sim$ Erlang truncated exponential $(\lambda, \theta)$

$$
\overline{\mathrm{H}}(\mathrm{y})=e^{-\theta x\left(1-e^{-\lambda}\right)}
$$

$$
\begin{aligned}
& P\left(\sum_{t=1}^{k} X_{t}<Y\right)=\int_{0}^{\infty} g_{k}(x) \bar{H}(x) d x \\
& =\int_{0}^{\infty} g_{k}(x) e^{-\theta x\left(1-e^{-\lambda}\right)} d x \\
& =\left[g *\left(\theta\left(1-e^{-\lambda}\right)\right)\right]^{k} \\
& \quad \mathrm{~S}(\mathrm{t})=\mathrm{P}[\mathrm{T}>\mathrm{t}] \\
& =\sum_{\mathrm{k}=0}^{\infty} \operatorname{Pr}\{\text { there are exactly } \mathrm{k} \text { contacts in }(0, \mathrm{t}]\} * \operatorname{Pr}\{\text { the cumulative total of antigenic diversity }<\mathrm{Y}\}
\end{aligned}
$$


$\therefore \mathrm{S}(\mathrm{t})=\sum_{\mathrm{K}=0}^{\infty} \mathrm{V}_{\mathrm{k}}(\mathrm{t}) \mathrm{P}\left[\sum_{\mathrm{i}=1}^{\mathrm{k}} \mathrm{X}_{\mathrm{i}}<\mathrm{Y}\right]$

$=\sum_{\mathrm{K}=0}^{\infty}\left[F_{\mathrm{k}}(\mathrm{t})-F_{\mathrm{k}+1}(\mathrm{t})\right]\left[g *\left(\theta\left(1-e^{-\lambda}\right)\right)\right]^{k}$

$L(t)=1-S(t)$

$L(t)=1-\sum_{\mathrm{K}=0}^{\infty}\left[F_{\mathrm{k}}(\mathrm{t})-F_{\mathrm{k}+1}(\mathrm{t})\right]\left[g *\left(\theta\left(1-e^{-\lambda}\right)\right)\right]^{k}$

$L(t)=\left[1-g^{*}\left(\theta\left(1-e^{-\lambda}\right)\right)\right] \sum_{k=1}^{\infty} F_{k}(t)\left[g^{*}\left(\theta\left(1-e^{-\lambda}\right)\right)\right]^{k-1}$

Let $\mathrm{U}_{1}, \mathrm{U}_{2} \ldots \mathrm{U}_{\mathrm{k}}$ represent the inter-arrival times between successive contacts which are correlated. Gurland (1995) has derived the cumulative distribution function of the sum, say $Z_{k}=\sum_{i=1}^{k} U_{i}$, when $U_{i}^{\prime s}$ from a order of exchangeable random variables are constantly correlated and each having exponential distribution and its p.d.f is given by

$$
f(u)=c e^{-u c}, c>0,0<u<\infty
$$

Such that the correlation co-efficient between $U_{i}$ and $U_{j}(\mathrm{i} \neq \mathrm{j})$ is $\rho$.

The c.d.f of $U$ is given by

$$
\begin{aligned}
F_{k}(u) & =P_{r}\left[Z_{k} \leq u\right] \\
& =(1-\rho) \sum_{i=1}^{\infty} \frac{\left(\rho_{k}\right)^{i} \eta\left[k+i, \frac{u}{b}\right]}{[1-\rho+k \rho]^{i+1}(k+i-1) !} \quad \text {, where } b=(1-\rho) / c . \\
\eta(k, u) & =\int_{0}^{u} e^{-c} c^{k-1} d c
\end{aligned}
$$

The Laplace transformation of the probability density function of $Z_{k}$ is given by

$$
\begin{gathered}
f_{k}^{*}(s)=\frac{1}{(1+b s)^{k}\left[1+\frac{k \rho b s}{(1-\rho)(1+b s)}\right]} \\
L(t)=\left[1-g^{*}\left(\theta\left(1-e^{-\lambda}\right)\right)\right] \sum_{k=1}^{\infty} F_{k}(t)\left[g^{*}\left(\theta\left(1-e^{-\lambda}\right)\right)\right]^{k-1}
\end{gathered}
$$

The p.d.f of $L(t)$ is given by,

$$
l(t)=\left[1-g^{*}\left(\theta\left(1-e^{-\lambda}\right)\right)\right] \sum_{k=1}^{\infty}\left[g^{*}\left(\theta\left(1-e^{-\lambda}\right)\right)\right]^{k-1} f_{k}^{*}(t)
$$

Using Laplace stieltjes transform of $l(t)$ is

$$
l^{*}(s)=\left[1-g^{*}\left(\theta\left(1-e^{-\lambda}\right)\right)\right] \sum_{k=1}^{\infty}\left[g^{*}\left(\theta\left(1-e^{-\lambda}\right)\right)\right]^{k-1} f_{k}^{*}(s) .
$$

Substituting (4.1) in (4.2), we get 
$l^{*}(s)=\left[1-g^{*}\left(\theta\left(1-e^{-\lambda}\right)\right)\right] \sum_{k=1}^{\infty}\left[g^{*}\left(\theta\left(1-e^{-\lambda}\right)\right)\right]^{k-1}\left[\frac{1}{(1+b s)^{k}\left[1+\frac{k \rho b s}{(1-\rho)(1+b s)}\right]}\right]$

To find the mean and variance time to zero conversion is

$$
\begin{aligned}
& f_{k}^{* \prime}(0)=-\left.\frac{d l^{*}(s)}{d s}\right|_{S=0} \\
& \text { and } \quad V(T)=E\left(T^{2}\right)-[E(T)]^{2} \\
& \text { where } f_{k}^{* \prime \prime}(0)=\left.\frac{d^{2} l^{*}(s)}{d s^{2}}\right|_{S=0} \\
& E(T)=\left[1-g^{*}\left(\theta\left(1-e^{-\lambda}\right)\right)\right] \sum_{k=1}^{\infty} f_{k}^{* \prime}(0)\left[g^{*}\left(\theta\left(1-e^{-\lambda}\right)\right)\right]^{k-1} \\
& E\left(T^{2}\right)=\left[1-g^{*}\left(\theta\left(1-e^{-\lambda}\right)\right)\right] \sum_{k=1}^{\infty} f_{k}^{* \prime \prime}(0)\left[g^{*}\left(\theta\left(1-e^{-\lambda}\right)\right)\right]^{k-1}
\end{aligned}
$$

Now

$$
\begin{aligned}
& f_{k}^{*}(s)=\frac{1}{(1+b s)^{k}\left[1+\frac{k \rho b s}{(1-\rho)(1+b s)}\right]} \\
& f_{k}^{*}(s)=(1+b s)^{1-k}\left[1+b s+\frac{k \rho s}{c}\right]^{-1} \quad(\because \text { on Simplification }) \\
& \left.f_{k}^{* \prime}(s)\right|_{S=0}=-\frac{d}{d s}\left[(1+b s)^{1-k}\left[1+b s+\frac{k \rho s}{c}\right]^{-1}\right] \\
& \left.f_{k}^{* \prime}(s)\right|_{S=0}=-\left.\left\{(1+b s)^{1-k} \frac{d}{d s}\left[1+b s+\frac{k \rho s}{c}\right]^{-1}+\left[1+b s+\frac{k \rho s}{c}\right]^{-1} \frac{d}{d s}[1+b s]^{1-k}\right\}\right|_{S=0} \\
& =-\left.(1+b s)^{-k}\left[-\frac{k \rho}{c}\left(1+b s+\frac{k \rho s}{c}\right)^{-2}-\left(1+b s+\frac{k \rho s}{c}\right)^{-k} b k\right]\right|_{S=0} \\
& =\frac{k \rho}{c}+b k \quad \because(1-\rho) / c=b \\
& f_{k}^{* \prime}(0)=\frac{k}{c}
\end{aligned}
$$




$$
\begin{aligned}
& \text { Alsofind }\left.\quad f_{k}^{* "}(s)\right|_{S=0} \\
& \left.f_{k}^{* \prime \prime}(s)\right|_{S=0}=\left.\frac{d}{d s} f_{k}^{* \prime}(s)\right|_{S=0} \\
& =\left.\frac{d}{d s}\left\{-(1+b s)^{-k}\left[\frac{k \rho}{c}\left(1+b s+\frac{k \rho s}{c}\right)^{-2}+b k\left(1+b s+\frac{k \rho s}{c}\right)^{-1}\right]\right\}\right|_{S=0} \\
& \left.f_{k}^{\prime \prime}(s)\right|_{S=0}=-\left\{\left.\begin{array}{l}
(1+b s)^{-k}\left[\frac{k \rho}{c}(-2)\left(1+b s+\frac{k \rho s}{c}\right)^{-3}+b k(-1)\left(1+b s+\frac{k \rho s}{c}\right)^{-2}\left(0+b+\frac{k \rho}{c}\right)\right] \\
+(1+b s)^{-k-1}(-b k)\left[\frac{k \rho}{c}\left(1+b s+\frac{k \rho s}{c}\right)^{-2}+b k\left(1+b s+\frac{k \rho s}{c}\right)^{-1}\right]
\end{array}\right|_{S=0}\right. \\
& =\left\{-\frac{2 k \rho}{c}\left(b+\frac{k \rho}{c}\right)-b k\left(b k+\frac{k \rho}{c}\right)-b k \cdot \frac{k \rho}{c}+b k\right\} \quad(\because 1-\rho=b c) \\
& f_{k}^{* \prime \prime}(0)=\frac{k\left(1-\rho^{2}\right)+k^{2}\left(1+\rho^{2}\right)}{c^{2}} .
\end{aligned}
$$

$u \sin g f_{k}^{* '}(0)$ in $E(T)$ then,

$$
E(T)=\left[1-g^{*}\left(1-e^{-\lambda}\right)\right] \sum_{k=1}^{\infty}\left(-\frac{k}{c}\right)\left[g^{*}\left(\theta\left(1-e^{-\lambda}\right)\right)\right]{ }^{k-1} \ldots
$$

Since $g(\cdot) \sim \exp (\mu)$, so that

$$
\begin{aligned}
g^{*}\left(\theta\left(1-e^{-\lambda}\right)\right) & =\frac{\mu}{\mu+\theta}-\frac{\mu}{\mu+\theta e^{-\lambda}} \\
E(T) & =\left[1-\frac{\mu}{\mu+\theta}+\frac{\mu}{\mu+\theta e^{-\lambda}}\right]\left[\sum_{k=1}^{\infty}\left(+\frac{k}{c}\right)\left[\frac{\mu}{\mu+\theta}+\frac{\mu}{\mu+\theta e^{-\lambda}}\right]^{k-1}\right]
\end{aligned}
$$




$$
\begin{aligned}
E(T) & =\left[1-\frac{\mu}{\mu+\theta}+\frac{\mu}{\mu+\theta e^{-\lambda}}\right]\left[+\frac{1}{c}\left(1-\left[\frac{\mu}{\mu+\theta}+\frac{\mu}{\mu+\theta e^{-\lambda}}\right]\right)^{-2}\right] \\
& =\frac{1}{c}\left[1-\frac{\mu}{\mu+\theta}+\frac{\mu}{\mu+\theta e^{-\lambda}}\right] * \frac{1}{\left[1-\frac{\mu}{\mu+\theta}+\frac{\mu}{\mu+\theta e^{-\lambda}}\right]^{2}} \\
& =\frac{1}{c} * \frac{1}{\left[1-\frac{\mu}{\mu+\theta}+\frac{\mu}{\mu+\theta e^{-\lambda}}\right]} \\
E(T) & =\frac{1}{c} \frac{(\mu+\theta)\left(\mu+\theta e^{-\lambda}\right)}{\left(\mu^{2}+2 \mu \theta+\theta^{2} e^{-\lambda}\right)}
\end{aligned}
$$

Using $f_{k}^{* "}(0)$ in $E\left(T^{2}\right)$

$$
E\left(T^{2}\right)=\left[1-g^{*}\left(\theta\left(1-e^{-\lambda}\right)\right)\right] \sum_{k=1}^{\infty}\left[\frac{k\left(1-\rho^{2}\right)+k^{2}\left(1+\rho^{2}\right)}{c^{2}}\right]\left[g^{*}\left(\theta\left(1-e^{-\lambda}\right)\right)\right]^{k-1}
$$

If $g(\cdot) \sim \exp (\mu)$, then,

$$
\begin{aligned}
g^{*}\left(\theta\left(1-e^{-\lambda}\right)\right) & =\frac{\mu}{\mu+\theta}-\frac{\mu}{\mu+\theta e^{-\lambda}} \\
& =\left[1-\frac{\mu}{\mu+\theta}+\frac{\mu}{\mu+\theta e^{-\lambda}}\right]\left[\frac{1}{c^{2}} \sum_{k=1}^{\infty}\left[k\left(1-\rho^{2}\right)+k^{2}\left(1+\rho^{2}\right)\right]\left[\frac{\mu}{\mu+\theta}-\frac{\mu}{\mu+\theta e^{-\lambda}}\right]^{k-1}\right. \\
& =\frac{\theta}{\mu+\theta}+\frac{\mu}{\mu+\theta e^{-\lambda}} * \frac{1}{c^{2}}\left\{\left(1-\rho^{2}\right) \sum_{k=1}^{\infty} k\left[\frac{\mu}{\mu+\theta}-\frac{\mu}{\mu+\theta e^{-\lambda}}\right]^{k-1}+\left(1+\rho^{2}\right) \sum_{k=}^{\infty} k^{2}\left[\frac{\mu}{\mu+\theta}-\frac{\mu}{\mu+\theta e^{-\lambda}}\right]^{k-1}\right\}
\end{aligned}
$$




$$
\begin{aligned}
& E\left(T^{2}\right)=\frac{1}{c^{2}} \frac{\mu \theta+\theta^{2} e^{-\lambda}+\mu^{2}+\mu \theta}{(\mu+\theta)\left(\mu+\theta e^{-\lambda}\right)} * \frac{(\mu+\theta)^{2}\left(\mu+\theta e^{-\lambda}\right)^{2}}{\left(\mu^{2}+2 \mu \theta+\theta^{2} e^{-\lambda}\right)^{2}}\left[\left(1-\rho^{2}\right)+\left(1+\rho^{2}\right) \frac{\left[\mu^{2}+2 \mu \theta e^{-\lambda}+\theta^{2} e^{-\lambda}\right]}{\left[\mu^{2}+2 \mu \theta+\theta^{2} e^{-\lambda}\right]}\right] \\
& V(T)=E\left(T^{2}\right)-[E(T)]^{2} \\
& \left.V(T)=\frac{1}{c^{2}} \frac{(\mu+\theta)^{2}\left(\mu+\theta e^{-\lambda}\right)^{2}}{\left(\mu^{2}+2 \mu \theta+\theta^{2} e^{-\lambda}\right)^{2}} * \frac{\mu^{2}+2 \mu \theta+\theta^{2} e^{-\lambda}}{(\mu+\theta)\left(\mu+\theta e^{-\lambda}\right)} \frac{2\left(\mu^{2}+\mu \theta+\theta^{2} e^{-\lambda}+\mu \theta e^{-\lambda}+\mu \theta \rho^{2}+\mu \theta e^{-\lambda} \rho^{2}\right)}{\left(\mu^{2}+2 \mu \theta+\theta^{2} e^{-\lambda}\right)}-1\right\} \\
& V(T)=\frac{(\mu+\theta)^{2}\left(\mu+\theta e^{-\lambda}\right)^{2}}{c^{2}\left(\mu^{2}+2 \mu \theta+\theta^{2} e^{-\lambda}\right)^{2}}\left[\frac{2(\mu+\theta)\left(\mu+\theta e^{-\lambda}\right)-2 \mu \theta \rho^{2}+2 \mu \theta e^{-\lambda} \rho^{2}}{(\mu+\theta)\left(\mu+\theta e^{-\lambda}\right)}-1\right] \ldots \ldots \ldots \ldots . . .(4.7)
\end{aligned}
$$

It may observed that the expected time to seroconversion remains unaffected even if the inter-contact times are correlated but the variance is the function of $\rho$, If we put $\rho=0$ in equation (4.7), the expression for the variance insides with that of the results in uncorrelated case obtained by Kannan and Parthasarathi (2017).

\section{NUMERICAL ILLUSTRATIONS}

\begin{tabular}{|c|c|c|}
\hline \multicolumn{3}{|c|}{ Table - 1 } \\
\hline \multicolumn{3}{|c|}{$\boldsymbol{\vartheta}=\mathbf{0 . 2}, \mu=\mathbf{0 . 2 ,} \lambda=\mathbf{0 . 6 ,}$} \\
$\boldsymbol{|}=\mathbf{0 . 3}$ \\
\hline$C$ & Mean & Variance \\
\hline 1 & 0.8729 & 0.7419 \\
\hline 2 & 0.4364 & 0.1855 \\
\hline 3 & 0.291 & 0.0824 \\
\hline 4 & 0.2182 & 0.0464 \\
\hline 5 & 0.1746 & 0.0297 \\
\hline 6 & 0.1455 & 0.0206 \\
\hline 7 & 0.1247 & 0.0151 \\
\hline 8 & 0.1091 & 0.0116 \\
\hline 9 & 0.097 & 0.0092 \\
\hline 10 & 0.0873 & 0.0074 \\
\hline
\end{tabular}

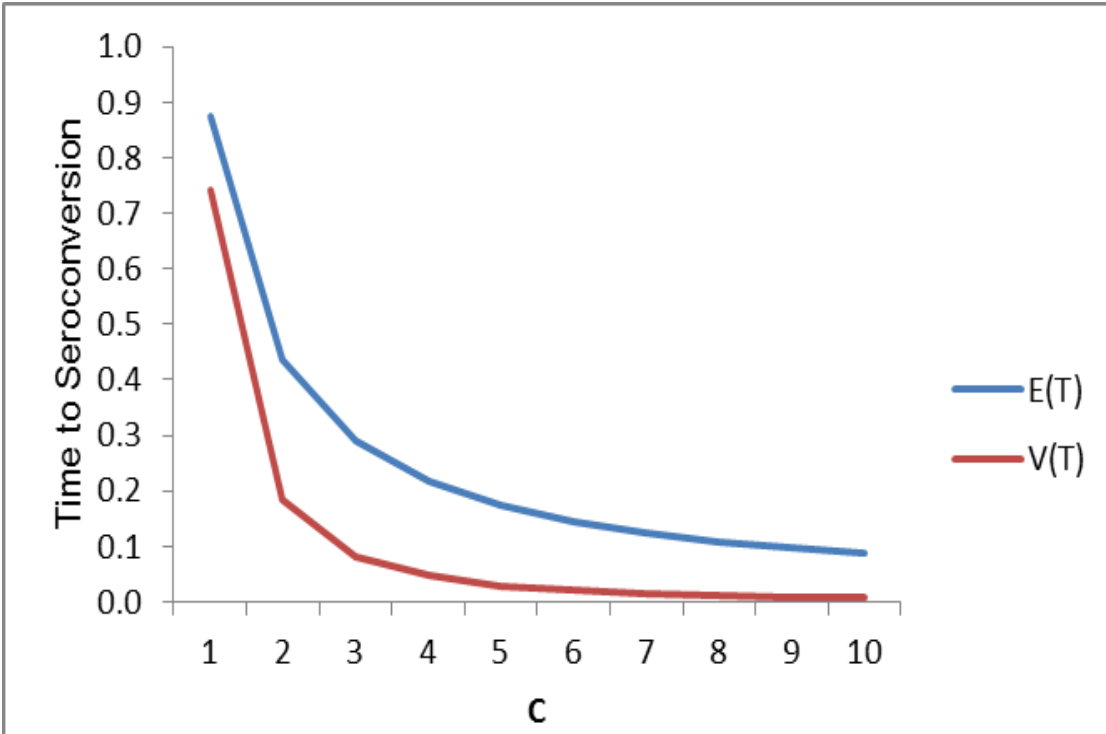

Figure -1 


\begin{tabular}{|c|c|c|}
\hline \multicolumn{3}{|c|}{ Table - 2} \\
\hline \multicolumn{3}{|c|}{$\begin{array}{c}\vartheta=0.2, \quad \lambda=0.6 \\
c=2, \rho=0.3\end{array}$} \\
\hline$\mu$ & Mean & Variance \\
\hline 0.1 & 0.4455 & 0.1941 \\
\hline 0.2 & 0.447 & 0.1956 \\
\hline 0.3 & 0.4496 & 0.1981 \\
\hline 0.4 & 0.4543 & 0.2026 \\
\hline 0.5 & 0.4585 & 0.2068 \\
\hline 0.6 & 0.4622 & 0.2105 \\
\hline 0.7 & 0.4653 & 0.2136 \\
\hline 0.8 & 0.468 & 0.2163 \\
\hline 0.9 & 0.4703 & 0.2187 \\
\hline 1 & 0.4724 & 0.2208 \\
\hline
\end{tabular}

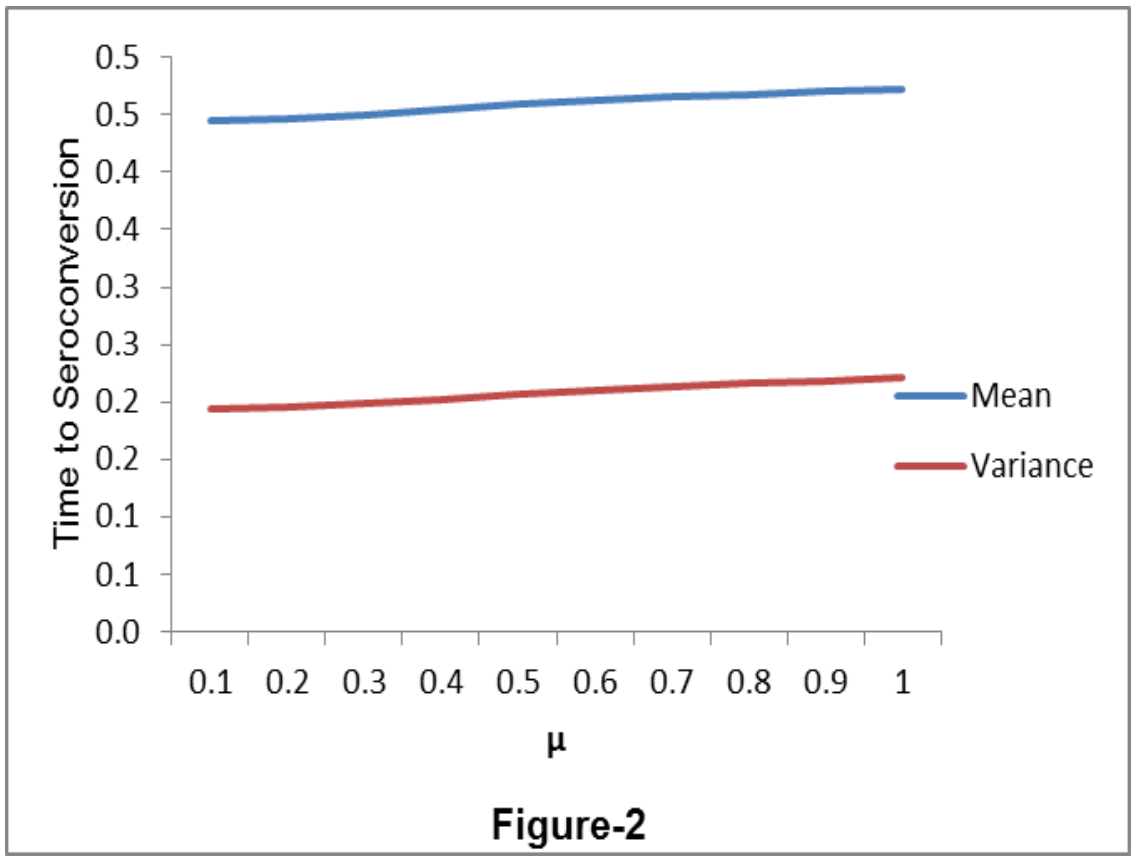

\begin{tabular}{|c|c|c|}
\hline \multicolumn{3}{|c|}{ Table-3 } \\
\hline \multicolumn{3}{|c|}{$\boldsymbol{\mu}=\mathbf{0 . 2}, \quad \lambda=\mathbf{0 . 6}$} \\
\hline $\boldsymbol{3}=\mathbf{2} \boldsymbol{\rho}=\mathbf{0 . 3}$ \\
\hline 0.01 & 0.4898 & 0.2390 \\
\hline 0.02 & 0.4813 & 0.2300 \\
\hline 0.03 & 0.4742 & 0.2227 \\
\hline 0.04 & 0.4683 & 0.2166 \\
\hline 0.05 & 0.4632 & 0.2115 \\
\hline 0.06 & 0.4590 & 0.2073 \\
\hline 0.07 & 0.4553 & 0.2037 \\
\hline 0.08 & 0.4522 & 0.2006 \\
\hline 0.09 & 0.4495 & 0.1980 \\
\hline 0.1 & 0.4472 & 0.1958 \\
\hline
\end{tabular}

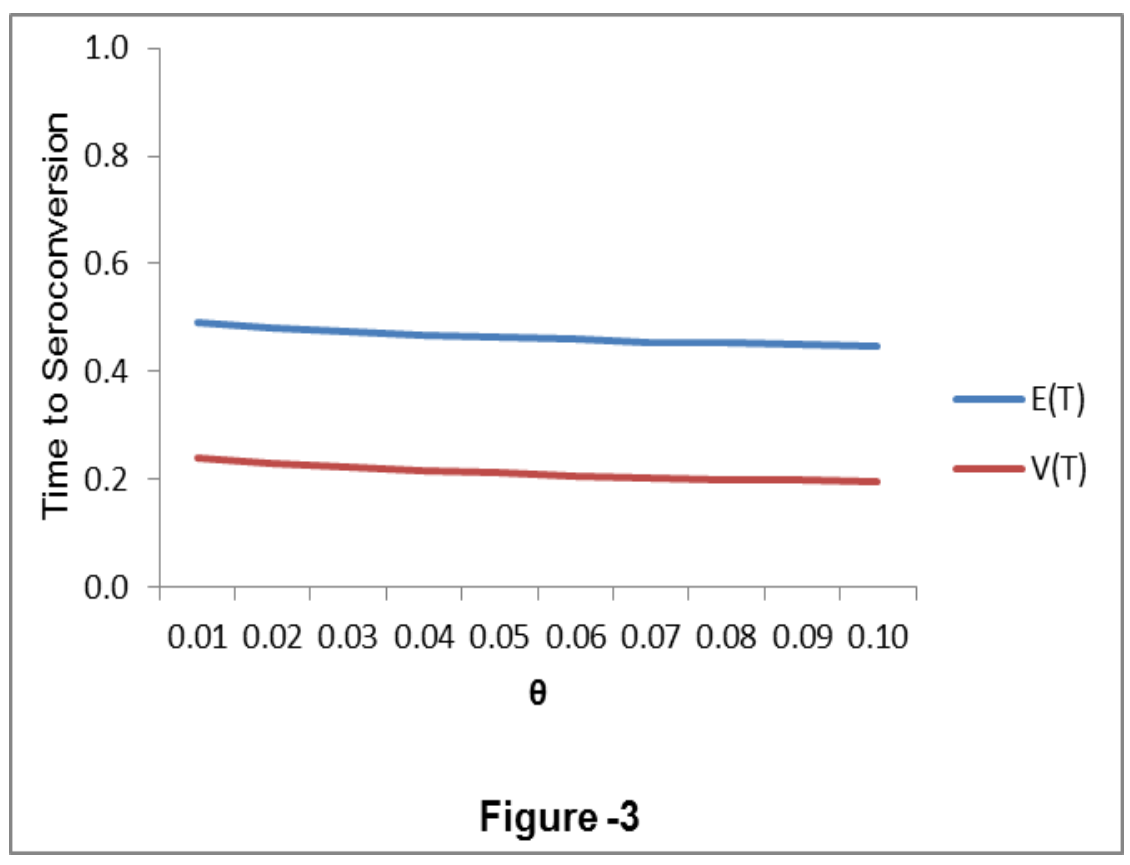




\begin{tabular}{|c|c|c|}
\hline \multicolumn{3}{|c|}{ Table-4 } \\
\hline \multicolumn{3}{|c|}{$\boldsymbol{\mu}=\mathbf{0 . 2 , \quad \vartheta = 0 . 5}$} \\
$c=2, \rho=0.3$ \\
\hline$\lambda$ & Mean & Variance \\
\hline 0.10 & 0.2366 & 0.0547 \\
\hline 0.20 & 0.1121 & 0.0121 \\
\hline 0.30 & 0.0787 & 0.0058 \\
\hline 0.40 & 0.0687 & 0.0044 \\
\hline 0.50 & 0.0684 & 0.0043 \\
\hline 0.60 & 0.0739 & 0.005 \\
\hline 0.70 & 0.0841 & 0.0065 \\
\hline 0.80 & 0.0986 & 0.0089 \\
\hline 0.90 & 0.1172 & 0.0125 \\
\hline 1.00 & 0.1396 & 0.0178 \\
\hline
\end{tabular}

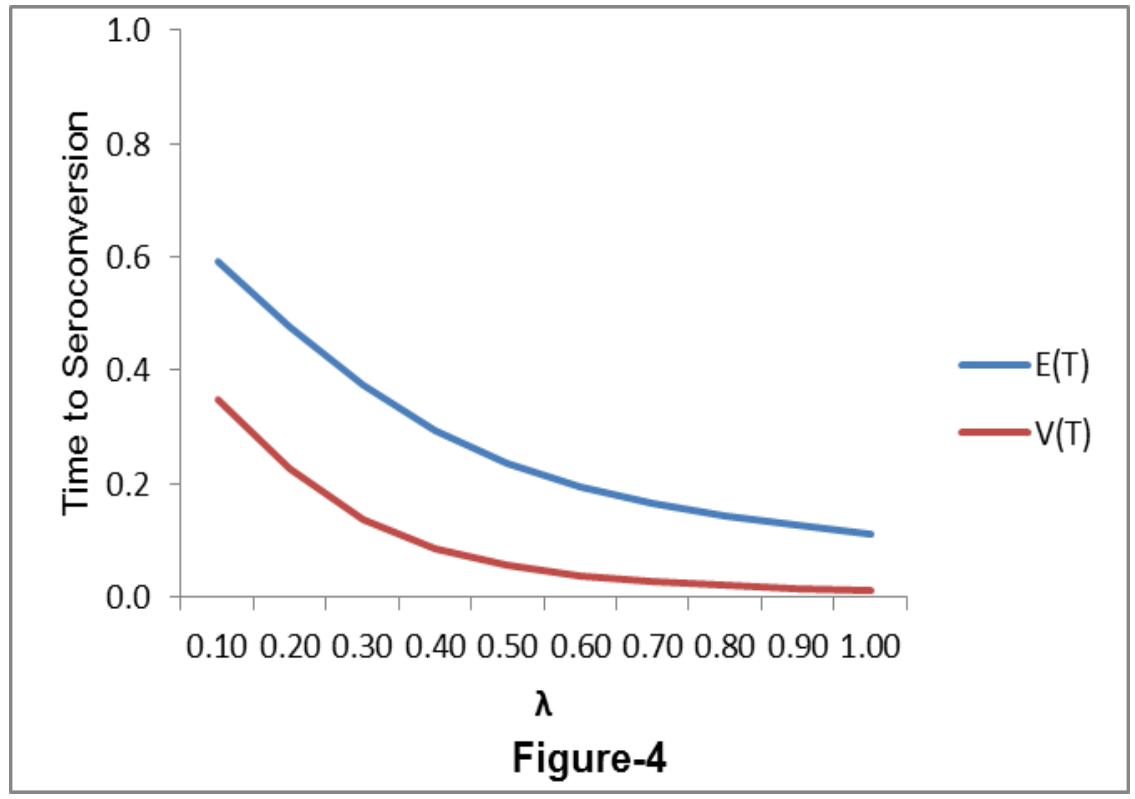

\begin{tabular}{|c|c|}
\hline \multicolumn{2}{|c|}{ Table $-\mathbf{5}$} \\
\hline \multicolumn{2}{|c|}{$\boldsymbol{\mu}=\mathbf{0 . 2}, \quad \boldsymbol{\vartheta}=\mathbf{0 . 5}$} \\
\hline $\boldsymbol{\rho}$ & $\mathrm{V}=\mathbf{0 . 3}$ \\
\hline 0.1 & 0.2161 \\
\hline 0.2 & 0.2151 \\
\hline 0.3 & 0.2135 \\
\hline 0.4 & 0.2112 \\
\hline 0.5 & 0.2083 \\
\hline 0.6 & 0.2047 \\
\hline 0.7 & 0.2005 \\
\hline 0.8 & 0.1957 \\
\hline 0.9 & 0.1902 \\
\hline 1 & 0.184 \\
\hline
\end{tabular}

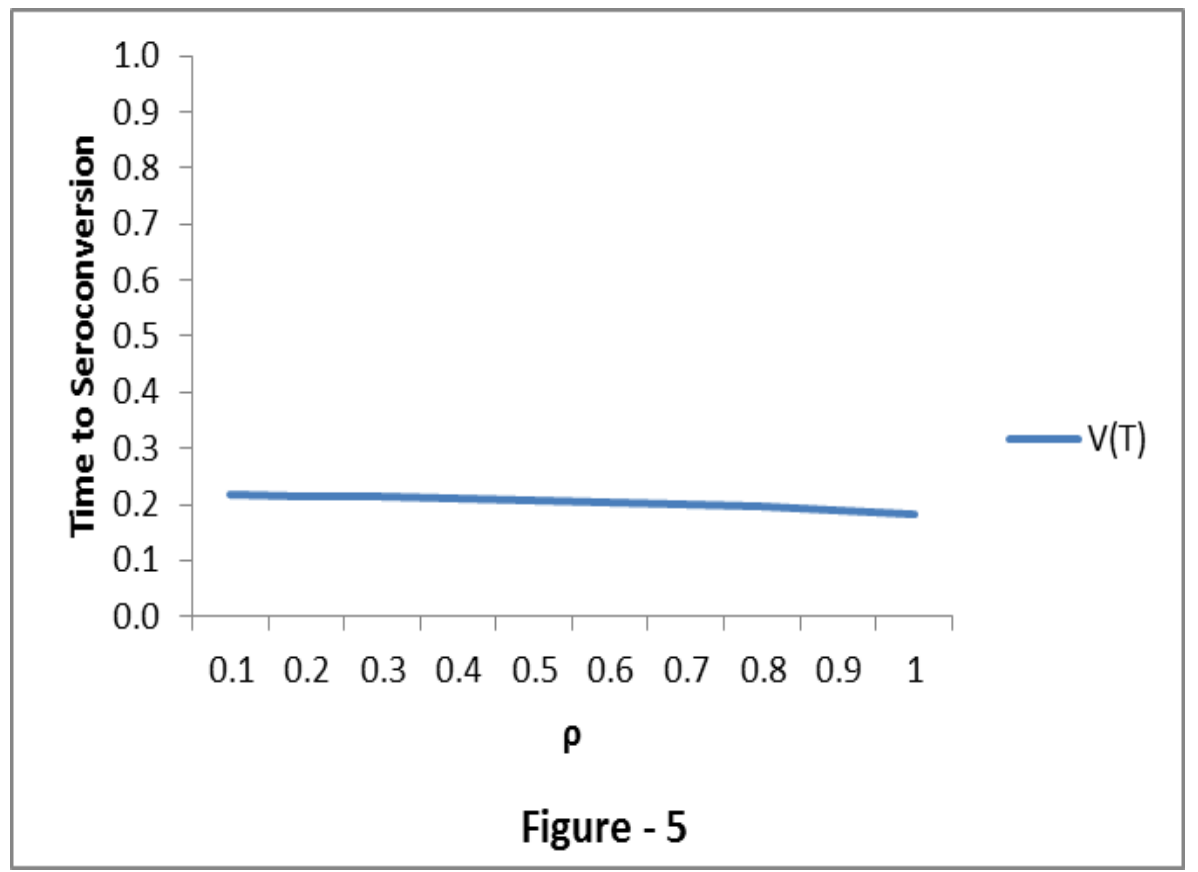

From the Table 1, we observed that for fixed $\theta, \mu, \lambda, \rho$ when $\mathrm{c}$ is the parameter of the inter-arrival time between contact increases which means average inter-arrival time become smaller, so the mean time to seroconversion decreases and also the variance time to seroconversion decreases. There is no impact of $\rho$ on the mean time to seroconversion and is presented in Figure.1.

From the Table 2, we observe that for fixed $\mathrm{c}, \theta, \lambda, \rho$, when $\mu$, the parameter of the random variable denoting Xi contributing to the antigenic diversity increases then it is seen that mean time to seroconversion and variance time to seroconversion increases as indicated in Table 2 and is also shown in Figure.2. 
From the Table 3, we observe that for fixed $c, \mu, \lambda, \rho$, when $\theta$, the parameter of the threshold distribution increases the mean time to seroconversions as well as the variance time to seroconversion decreases. Figure. 3 shows the graph.

From the Table 4, we observe that for fixed $c, \mu, \theta, \rho$, when $\lambda$, the parameter of the threshold distribution increases the mean time to seroconversions decreases, the variance time to seroconversion decreases. Figure. 4 shows the graph.

From the Table 5, we observe that for fixed $c, \mu, \theta, \lambda$, when $\rho$, the constant correlation between successive contacts increases, the variance time to seroconversion decreases as indicated in Figure 5.

\section{REFERENCES}

[1] Esary, J.D.,A. W. Marshall and F. Proschan (1973). Shock model and wear processes.Ann. Probability,1(4), 627-649.

[2] Gurland, J. (1955). Distribution of maximum of the arithmetic mean of correlation random vriables. Ann. Mathematical Statistics, 26,294-300.

[3] Kannan,R.,Vanimalini.R and RSathiyamoorthi (2012). A Stochastic Model With Antigenic Diversity Threshold Of Hiv Transmission Under Correlated Intercontact Times

[4] Kannan, R.,Parthasarathi.G (2017). A stochastic model for the estimation of time to seroconversion of HIV transmission using Erlang Truncated Exponentail distribution. International Journal of Statistics and Applied Mathematics , 2(6), 211-215.

[5] Kannan,R and R. Sathiyamoorthy(2006). Stochastic Model for time to seroconversion of HIV transmission under correlated intercontact times, Annamalai University Science Journal, Vol.143,1-12.

[6] Nowak, M.A. and R.M. May (1991). Mathematical biology of HIV infection antigenic variation and diversity threshold. Mathematical Biosciences, 106,1-21.

[7] Sathiyamoorthi, R. (1979). Cumulative Damage Model with correlated Interarrival Times of Shocks IEEE. Transactions on Reliability, R 25, No.23.

[8] Stilianakis, N.D. Schenzle and K.Dietz (1994). On the antigenic diversity threshold model for AIDS. Mathematical Biosciences, $121,235-247$. 